Revue des patrimoines

Le cheval et ses patrimoines (1ère partie)

\title{
Le cheval et son cavalier dans la peinture murale des $\mathrm{XI}^{\mathrm{e}}$-XIII ${ }^{\mathrm{e}}$ siècles
}

\section{Christian Davy}

\section{OpenEdition}

\section{Journals}

\section{Édition électronique}

URL : http://journals.openedition.org/insitu/9724

DOI : 10.4000/insitu.9724

ISSN : 1630-7305

\section{Éditeur}

Ministère de la culture

\section{Référence électronique}

Christian Davy, "Le cheval et son cavalier dans la peinture murale des XIe-XIII siècles », In Situ [En ligne], 18 | 2012, mis en ligne le 31 juillet 2012, consulté le 19 avril 2019. URL : http:// journals.openedition.org/insitu/9724; DOI : 10.4000/insitu.9724

Ce document a été généré automatiquement le 19 avril 2019

\section{(c) $($ i) $(9)$}

In Situ Revues des patrimoines est mis à disposition selon les termes de la licence Creative Commons Attribution - Pas d'Utilisation Commerciale - Pas de Modification 4.0 International. 


\title{
Le cheval et son cavalier dans la peinture murale des $\mathrm{XI}^{\mathrm{e}}$-XIII ${ }^{\mathrm{e}}$ siècles
}

\author{
Christian Davy
}

L'invitation à présenter cette communication a permis à un étranger au monde équin d'observer sous un angle nouveau des images qu'il interroge habituellement de manière autre. L'auteur s'excuse donc auprès des spécialistes du cheval s'il arrive que l'historien de l'art énonce quelque évidence pour eux. Le récolement des scènes dans lesquelles interviennent un ou plusieurs chevaux, montés ou non, s'est avéré suffisamment riche pour lancer les bases d'une étude sur la place qu'occupent le cheval et son cavalier dans la peinture murale médiévale, plus particulièrement dans la production réalisée entre le $\mathrm{XI}^{\mathrm{e}}$ et le XIII ${ }^{e}$ siècle. Le temps imparti à cette approche n'a bien entendu pas permis la mise en place d'un corpus exhaustif. Cependant, les images sélectionnées sont suffisamment représentatives pour mettre en évidence des phénomènes éclairant la représentation sociale ou symbolique du cheval à cette époque. Leur répartition géographique entre la Normandie, le Maine, l'Anjou, la Touraine, le Poitou, le Berry, la Bourgogne, l'Auvergne ou le Roussillon autorise une réflexion au niveau du territoire français dans son acception actuelle.

2 Deux voies de recherche ressortent de l'examen préliminaire des images contenant le cheval et son cavalier. La première consiste à aborder l'image comme le reflet, voire le miroir, d'une réalité terrestre. Cependant, en remarques préliminaires, il est nécessaire de rappeler que la peinture murale médiévale est un art figuratif abstrait aux fonctions décoratives et signifiantes. En effet, l'image médiévale constitue un support de messages envoyés par le commanditaire à ses contemporains réunis au sein de groupes plus ou moins développés en nombre. Son caractère signifiant est multiple: les peintres emploient une écriture basée sur le signe comme convention qui change de sens en fonction du contexte dans lequel il est introduit. D'une manière concrète, la figuration d'un arbre peint isolément, comme par exemple dans la Chasse de saint Gilles au LorouxBottereau (Loire-Atlantique) à la fin du XII ${ }^{e}$ siècle (fig. $\mathbf{n}^{\circ} \mathbf{1}$ ), peut aussi bien signifier un arbre, un bosquet ou une forêt, soit l'individu ou l'ensemble, mais dans tous les cas, il signale que la scène dans laquelle il est inséré se déroule en extérieur. 
Figure 1

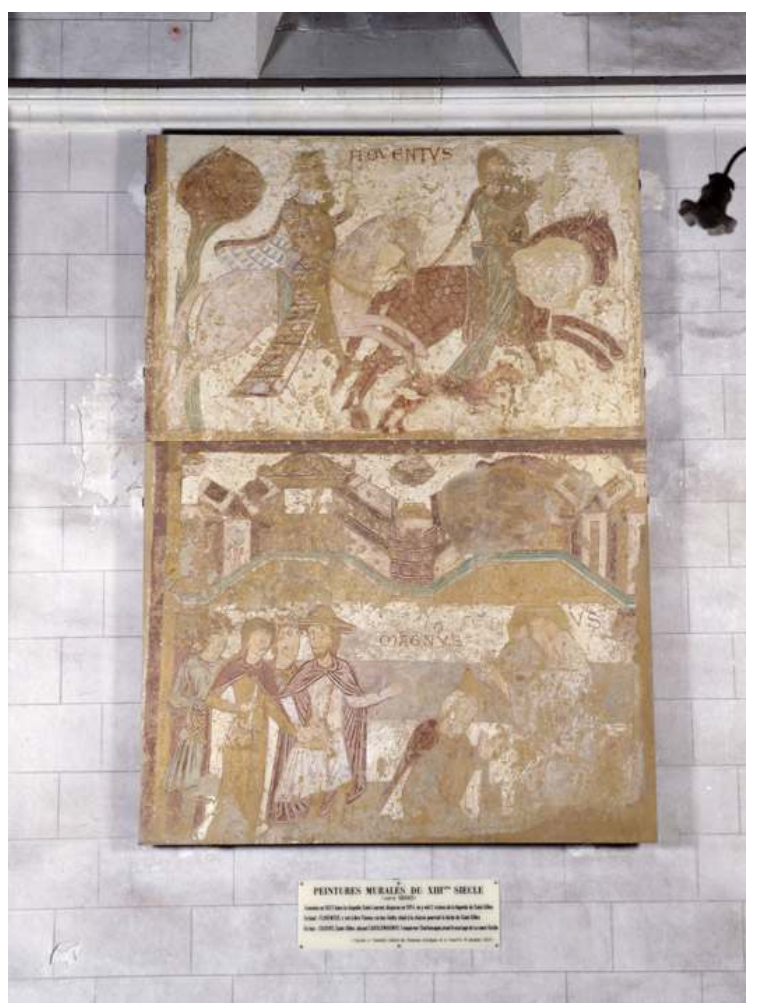

Le Loroux-Bottereau. La Chasse de saint Gilles, détail : la chevauchée des chasseurs.

Phot. Pillet, Denis. @ Conseil régional des Pays de la Loire, inventaire général, 1995.

Cette diversité de sens se rencontre de manière quasi systématique: en fonction des textes, le lion peut aussi bien être lu comme un symbole du Christ que celui du mal ${ }^{1}$, seule l'analyse du contexte permet alors d'orienter correctement la lecture de l'image. Il est également nécessaire de rappeler que toutes les images médiévales parvenues jusqu'à notre époque sont des œuvres usées et lacunaires - de plus, essentiellement conservées dans des édifices religieux - qui méritent une analyse critique avant toute interprétation. Un cycle dédié à saint Gilles a été peint à la fin du XII ${ }^{\mathrm{e}}$ siècle sur le mur nord de la nef de l'église paroissiale Notre-Dame à Thoiré-sur-Dinan (Sarthe). Les peintures murales, dont l'épisode de la chasse, ont été particulièrement maltraitées lors du percement d'une fenêtre à l'époque moderne. 
Figure 2

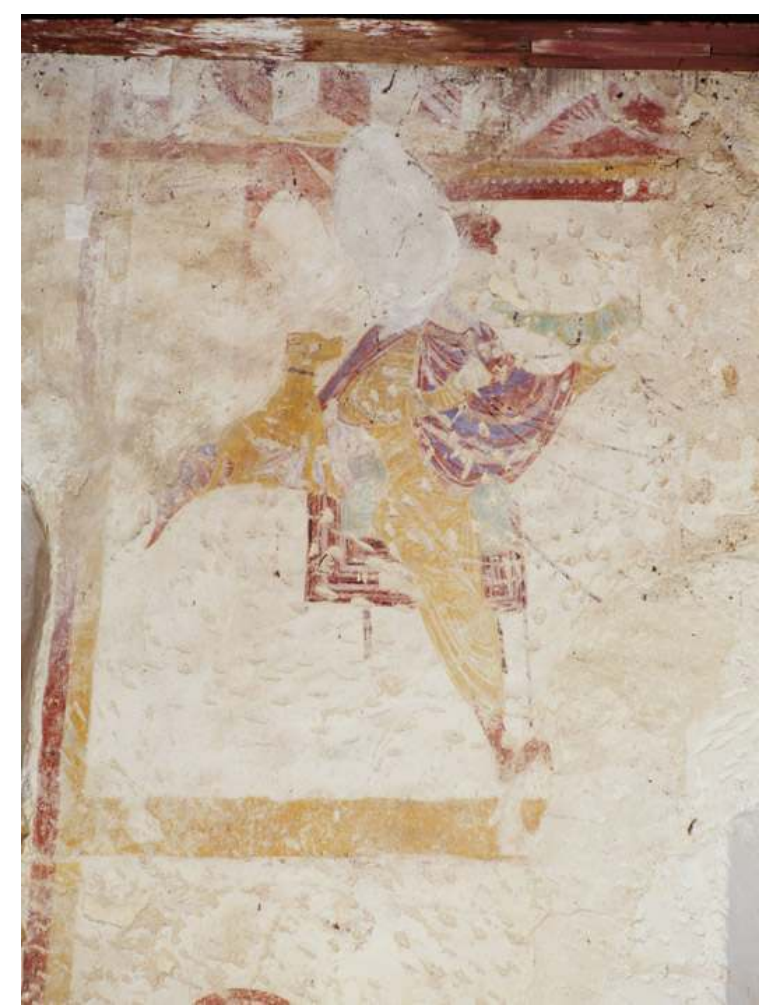

Thoiré-sur-Dinan. Église paroissiale, nef, mur nord. La Chasse de saint Gilles, détail : le cavalier sonnant I'hallali.

Phot. Giraud, Patrice. (c) Conseil régional des Pays de la Loire, inventaire général, 1995

4 L'identification du personnage flottant dans les airs comme un cavalier (fig. $\mathbf{n}^{\circ} \mathbf{2}$ ) s'effectue grâce à la position de l'homme et la reconnaissance des éléments de harnachement de cheval dont la figuration a entièrement disparu. En fait, le cavalier est assis sur une selle verte au troussequin plus développé que le pommeau. Celle-ci a été posée sur un tapis de selle attaché par deux sous-ventrières noires. D’autres lignes noires représentent la bricole, une partie des sangles du mors et les rênes tenues dans la main droite du cavalier. La tension de la jambe du cavalier donne l'emplacement de l'étrier qui n'est plus visible, comme la figuration-même du cheval. 


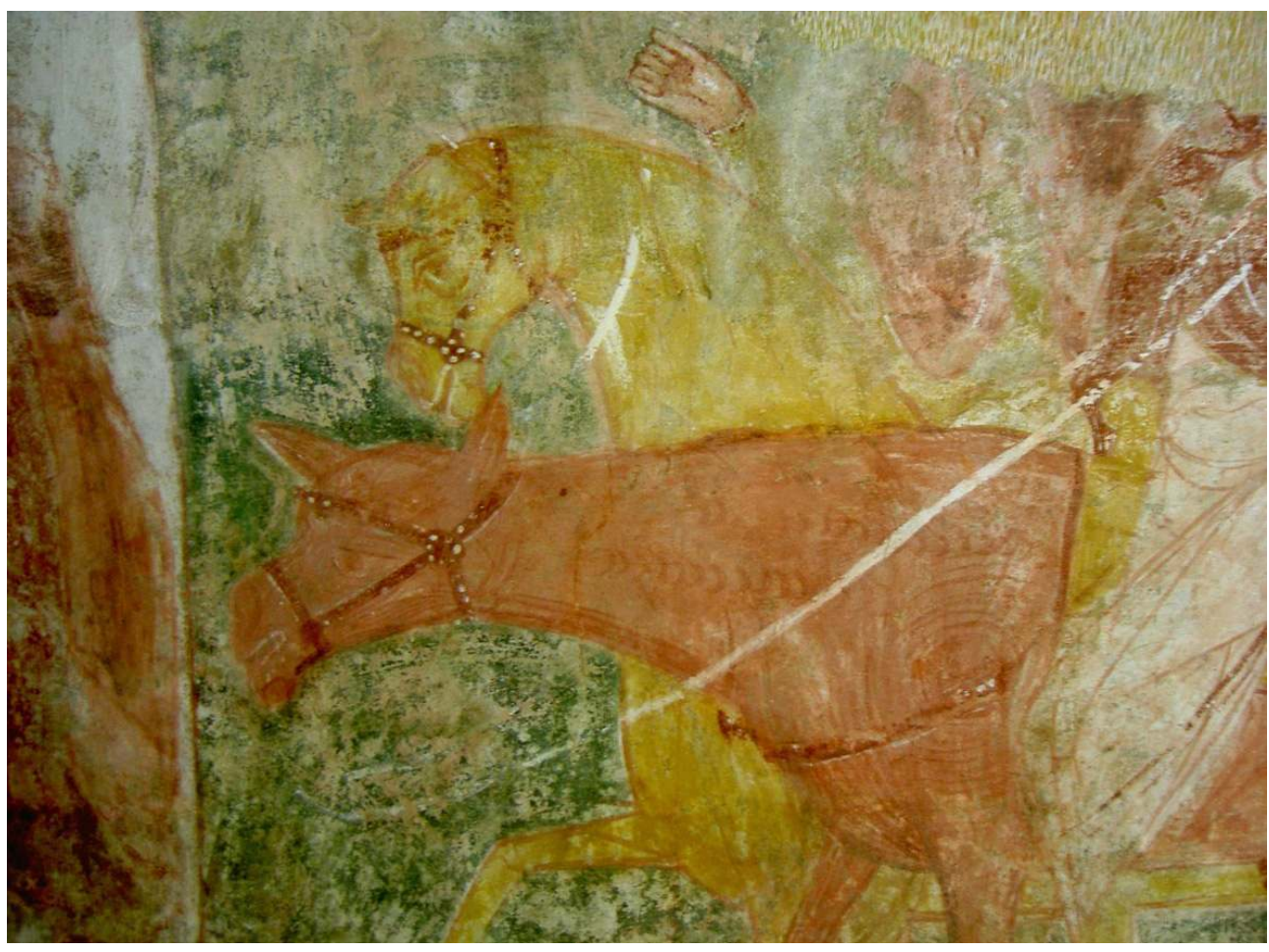

Saint-Savin-sur-Gartempe. Église abbatiale, nef, voûte. Joseph emporté en esclavage en Égypte, détail : les chevaux des marchands.

PHOT. DAVY, CHRISTIAN. (C) CHRISTIAN DAVY.

Dans le cas de la scène "Joseph emmené en esclavage en Égypte » peinte à la fin du $\mathrm{XI}^{\mathrm{e}}$ siècle à la voûte de la nef de l'église abbatiale de Saint-Savin-sur-Gartempe (Vienne), la peinture murale est mieux conservée. L'image (fig. $\mathbf{n}^{\circ} \mathbf{3}$ ) permet d'observer plusieurs détails du harnachement. La bricole et la croupière sont en place, mais la représentation leur donne-t-elle une tension correcte ? Les brides et les sangles de mors sont rehaussées de ponctuations blanches. S'agit-il de détails fonctionnels ou ornementaux? Le mors semble bien être placé trop en arrière de la bouche des chevaux. Est-ce une erreur de la part du peintre ou bien un artifice pour mieux faire ressortir le rehaut blanc qui éclaire les lèvres? Quelle valeur attribuer à la position des rênes dans les mains des marchands esclavagistes? Leur agencement est-il induit par une volonté décorative qui organise les lignes de la scène entre elles ou bien par une autre plus attentive à la réalité des mouvements? Ainsi, cette voie de recherche apparaît particulièrement délicate par une situation très variable entre la représentation fidèle d'une réalité terrestre et le geste artistique, voire symbolique. Toutefois, son exploration devrait être assurément fructueuse et elle mériterait la constitution d'un groupe de travail, réunissant notamment spécialistes du cheval, historiens, historiens de l'art, archéologues. Le deuxième axe de recherche est plus habituel pour l'historien de l'art. Il s'agit en effet d'analyser la place qu'occupent le cheval et son cavalier dans un corpus préalablement défini, avant de la resituer dans un contexte iconographique élargi et de la confronter à l'état de la connaissance sur la société qui a produit ces images. Il s'agit dans cette communication de pratiquer cet exercice dans un art, la peinture murale du Moyen Âge central, qui s'avère être un support de communication vers un large public, composé principalement par la masse des fidèles. En d'autres termes, quel message désirait faire passer le commanditaire 
lorsqu'il faisait représenter le cheval et son cavalier ? Est-il de nature positive, neutre, négative, discriminante, morale, homogène? Renvoie-t-il à des valeurs semblables à celles déterminées par l'historien Daniel Pichot à travers la lecture des textes manceaux ${ }^{2}$ ? En fin de compte, image et texte ont-ils la même portée sur ce thème précis? Cet historien part de la constatation du « simple fait d'avoir donné son nom au groupe social qui va devenir dominant chez les laïcs dit assez l'importance que l'animal a pu jouer » pour souligner que les textes présentent le cheval comme « une richesse qui circule et s'échange ", qu'il est l'« apanage d'une classe aisée ", qu'il en représente "le lien sinon exclusif, du moins fortement privilégié » et qu'il constitue un "élément indispensable dans la représentation sociale ». Il remarque, à la suite de Georges Duby, l'assimilation «entre le genre de vie réputé noble et l'usage du cheval». Nous allons voir que les diverses représentations en peinture murale confirment les conclusions apportées par l'étude des textes, et vont au-delà en donnant une portée morale ou eschatologique aux divers messages transmis.

\section{Le cheval, arme de guerre}

6 La guerre constitue l'activité primordiale des miles. Les lieux de culte tolèrent sur leurs murs et voûtes cette activité dans une forme acceptable par l'Église, telle la croisade menée pour la délivrance du tombeau du Christ. Paul Deschamps ${ }^{3}$ avait identifié en son temps la bataille contre Kerboga sous les murs d'Antioche peinte dans l'église paroissiale de Poncé-sur-le-Loir (Sarthe). Un cycle de trois scènes raconte la charge des croisés face aux assiégeants, la charge miraculeuse des saints Mercurius, Démétrius et Georges et la fuite de Kerboga, le chef des Sarrasins. Les chevaux sont représentés au galop dans un face à face parfaitement frontal. L'usure de la peinture a fait perdre le détail des représentations, mais la puissance des destriers est remarquablement plantée dans l'attitude des deux postérieurs au sol et les antérieurs levés et lancés vers l'avant. Cette position n'est pas naturelle, mais cette représentation est la conséquence des choix esthétiques du peintre. Il en est de même pour les robes des chevaux dont les couleurs foncées et claires ont été choisies en fonction de la meilleure lisibilité possible de l'image. Les chevaux clairs se détachent sur un fond sombre et inversement ${ }^{4}$. La disposition générale de ces alternances colorées permet d'attribuer des robes blanches au cheval de saint Georges et sans doute à ceux de ses compagnons, respectant ainsi le récit miraculeux de l'Histoire anonyme de la première croisade ${ }^{5}$. S'il est assuré qu'il s'agit ici de la bataille d'Antioche, en revanche les historiens débattent toujours de l'identification des batailles présentées sur la totalité du mur nord de la chapelle de la commanderie des Templiers de Cressac (Charente) ${ }^{6}$. Les deux registres présentent-ils une ou deux batailles ? De même, s'agit-il de la Croisade ou de la Reconquista? Les nombreux chevaux, parfois membrés, aux robes claires, foncées ou de ton moyen, sont présentés dans différentes positions : au pas à la sortie d'une ville fortifiée jusqu'à la charge au galop face à l'ennemi. Cette dernière position est semblable à celle précédemment remarquée à Poncé-sur-leLoir. Elle constitue en fait le stéréotype du galop à grande vitesse utilisé par les peintres. Ces chevaliers qui combattent pour le Christ constituent l'image terrestre des armées célestes. Saint Michel, chef des milices célestes, combat le dragon à la tête de ses troupes d'anges. L'épisode apocalyptique est peint au porche de l'abbatiale de Saint-Savin-surGartempe (Vienne) dans une version chevaleresque : Michel, à la tête de sa troupe, charge le dragon lance levée. La scène est lacunaire et usée. Cependant, il est possible de voir que 
la blancheur immaculée est attribuée à la robe de son cheval, tandis que la croupe évoquant le reste de la cavalerie est de couleur verte ${ }^{7}$. Au revers de la façade du même édifice, l'image de deux cavaliers chargeant l'un contre l'autre trouve son équivalent dans le bras nord du transept de l'église prieurale de Château-Gontier (Mayenne). Les deux images ont été peintes à la fin du XI ${ }^{\mathrm{e}}$ siècle. Elles témoignent à la fois de la nouveauté du duel à cheval et de la permanence du thème bien connu dans l'enluminure dès l'époque carolingienne qu'est le combat de deux hommes figurant le combat de l'âme, et de son adaptation à la société médiévale : le combattant à pied a été remplacé par le cavalier. Plus, les deux protagonistes chargent lance baissée, non plus projetée comme un javelot, innovation dans la technique de combat en cette fin de siècle. ${ }^{8}$. Le tournoi fut l'un des exutoires de la guerre. Les premières représentations en peinture murale conservées avaient été exécutées au milieu du XIII ${ }^{\mathrm{e}}$ siècle. Ce thème a été aussi bien peint dans des édifices laïcs que cultuels. L'église prieurale de La Ramée (Mayenne) conserve, malgré sa transformation en étable-grange, une scène de tournoi (fig. $\left.\mathbf{n}^{\circ} 4\right)\left(\right.$ fig. $\left.\mathbf{n}^{\circ}{ }^{\circ}\right)$ peinte sur le mur occidental de la nef. Le caparaçon des chevaux aux armes de leurs cavaliers témoigne de l'aspect ostentatoire donné à ce simulacre de guerre. Le thème du tournoi connaît un succès immense à travers l'ensemble du territoire français pendant les derniers siècles du Moyen Âge ${ }^{9}$.

Figure 4

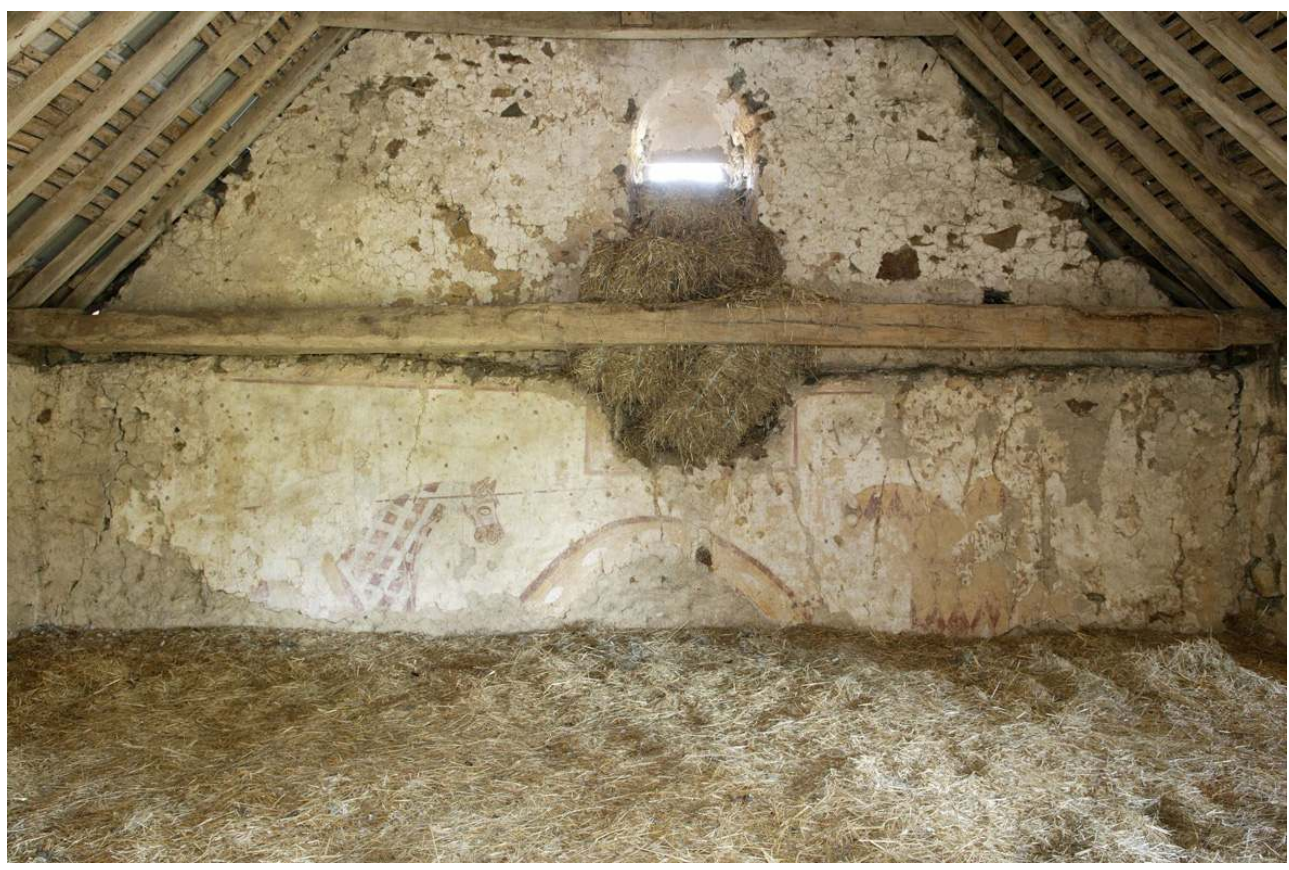

La Chapelle-Rainsouin, église prieurale de la Ramée, mur occidental. Tournoi entre deux chevaliers. Phot. Giraud, Patrice. (c) Conseil régional des Pays de la Loire, inventaire général, 2005. 


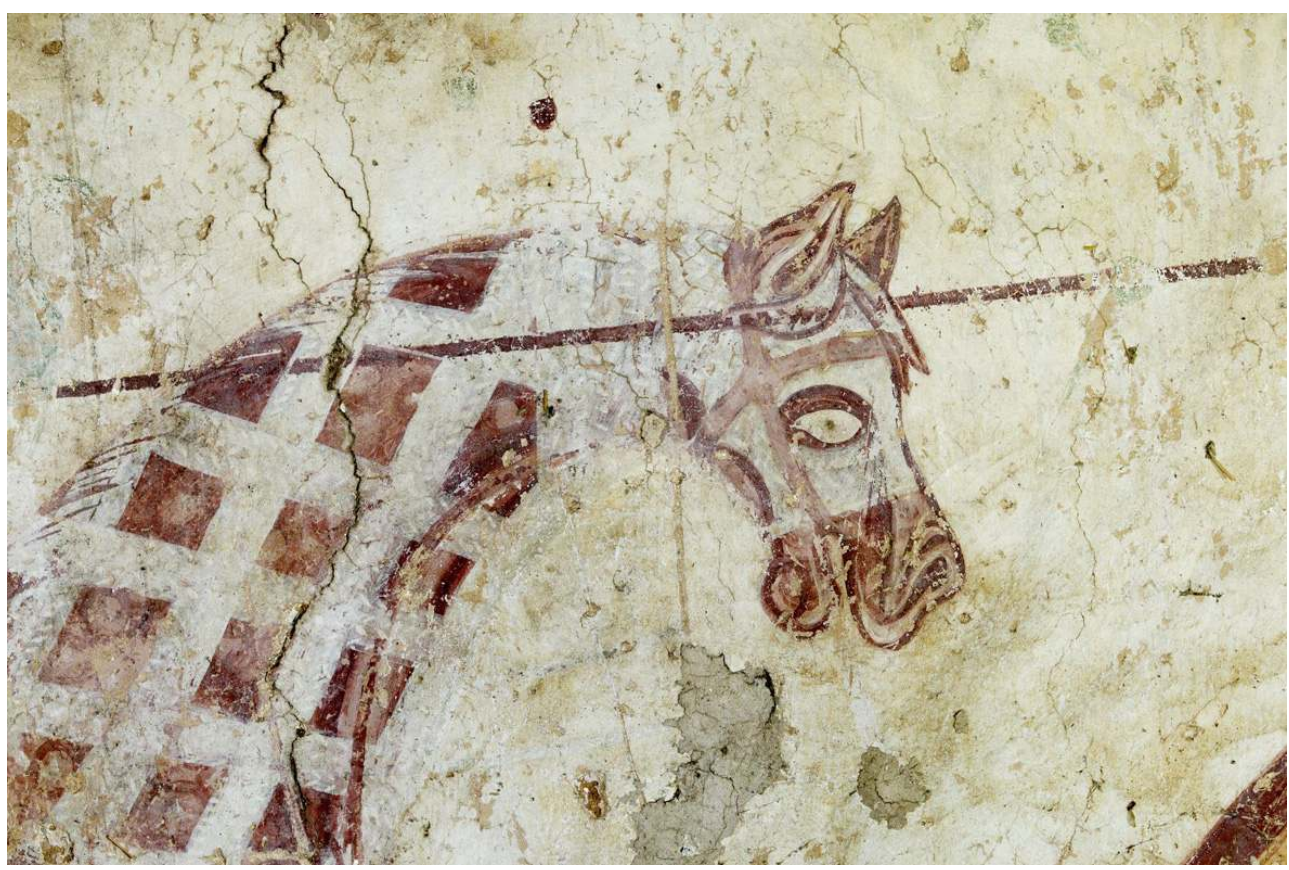

La Chapelle-Rainsouin, église prieurale de la Ramée, mur occidental. Tournoi entre deux chevaliers, détail : l'encolure du cheval gauche.

Phot. Giraud, Patrice. @ C Conseil régional des Pays de la Loire, inventaire général, 2005.

\section{Le cheval, la marque des puissants}

Les textes témoignent au détour des récits hagiographiques ou des chartes que le cheval est l'apanage des classes aisées, que ses membres soient évêque, abbé, clerc ou noble laïc, homme ou femme ${ }^{10}$. L'image en témoigne également. L'interprétation de l'épisode peint dans la chapelle troglodytique Saint-Jean, puis Sainte-Radegonde reste controversée ${ }^{11}$. S'agit-il d'un épisode lié à l'histoire de la sainte dédicataire ou bien à celle, plus romantique, d'Aliénor d'Aquitaine? Un couple royal, qui se reconnait aux couronnes et aux manteaux fourrés de vair, est accompagné d'un cavalier portant un faucon sur sa main gantée et de deux autres personnages sans attribut spécifique. Inégalement répartis dans la scène, les cinq personnages chevauchent tranquillement et conversent entre eux ${ }^{12}$ . La partie inférieure de la scène est détruite, mais il est possible de constater que l'allure des chevaux est paisible et que l'un d'eux marche assurément à l'amble. Sans écarter définitivement une fantaisie de la part du peintre, cette représentation pourrait témoigner du dressage effectué par l'homme sur le cheval pour améliorer le confort du cavalier lors de longs trajets ${ }^{13}$. Cette représentation d'allure n'est pas exceptionnelle, car elle a également été reproduite dans la Chevauchée des rois mages peinte au milieu du XII ${ }^{\mathrm{e}}$ siècle dans le cloître de l'abbaye Saint-Aubin d'Angers (Maine-et-Loire) ${ }^{14}$.

Le succès de la dévotion à saint Gilles autorise plusieurs représentations conservées de roi cavalier dans l'épisode de la Chasse de saint Gilles. La plus connue se trouve au LorouxBottereau (Loire-Atlantique) (voir fig. $\mathbf{n}^{\circ} \mathbf{1}$ ). L'image est inspirée du texte de Guillaume de Berneville rédigé en 1164. D'autres exemplaires, peints à la fin du XII et au cours du XIII ${ }^{e}$ siècle, sont visibles notamment aux Roches-l'Évêque, à Saint-Jacques-des-Guérets, à 
Huisseau-en-Beauce (tous trois en Loir-et-Cher) et à Thoiré-sur-Dinan (Sarthe). Au Loroux-Bottereau, la posture des chevaux adopte le stéréotype précédemment remarqué des animaux lancés au galop. Le peintre donne aussi cette position au chien de chasse courant entre les pattes du cheval de tête. La robe de ce dernier est brune et pommelée, tandis que l'usure ne permet plus de déterminer l'aspect de celle du cheval royal. Cependant, elle semble claire et rehaussée de bleu, couleur assurée de la crinière. Les rois cavaliers sont acteurs dans un autre thème qui eut un plus grand succès encore. SaintMartin de Fénollar (Pyrénées-Orientales), Angers (Maine-et-Loire), Brinay (Cher), PetitQuevilly (Seine-Maritime) et Château-Gontier (Mayenne), parmi les sites les plus connus, présentent une Chevauchée des rois mages peinte le plus souvent dans un cycle de la Nativité de l'époque romane. L'Adoration des rois mages peinte au XIII ${ }^{\mathrm{e}}$ siècle à Gargilesse (Indre) est complétée d'un épisode rare dans lequel l'écuyer, nommé Galopin, garde les chevaux pendant que les rois mages se prosternent au pied de la Vierge à l'Enfant. La plus ancienne peinture murale de cette chevauchée est conservée dans l'église pré-romane de Saint-Pierre-les-Églises (commune de Chauvigny, Vienne). Les mages sont coiffés d'une coiffure triangulaire, dite bonnet phrygien. Ce n'est que par la suite, à l'époque romane, qu'ils sont coiffés d'une couronne attestant de leur royauté nouvelle. Le programme peint sur l'arc triomphal de l'église prieurale de Vic (commune de Nohant-Vic, Indre) place le fidèle devant le balancement habituel entre les deux moments forts de l'année liturgique : Pâques avec la Déposition de croix et Noël avec l'Adoration des rois mages notamment. Cette dernière est précédée d'une chevauchée spectaculaire (fig. $\left.\mathbf{n}^{\circ} \mathbf{6}\right)$. Le peintre a figé les trois chevaux dans une posture identique : le membre antérieur gauche et l'arrière droit sont levés haut et l'ensemble penche vers l'avant dans une expression de grande tension. Il ne s'est manifestement pas attaché à matérialiser les harnachements. Seuls deux ou trois traits signalent une discrète selle sur laquelle est assis le roi mage situé au premier plan. La recherche d'un parti esthétique est ici assurée. Un cran dans la hiérarchie aristocratique est franchi avec la figuration de quatre cavaliers assimilés aux quatre premiers empereurs chrétiens au baptistère Saint-Jean de Poitiers (Vienne) ${ }^{15}$. Cette image peinte vers 1100 répond, avec son équivalent des années 1200 situé à Cressac, aux nombreuses statues équestres installées à l'époque romane sur les façades occidentales des églises du Poitou. Les empereurs ont adopté une posture différente sur leurs chevaux. Jusqu'à présent, le cavalier poussait sur les étriers, tandis qu'à Saint-Jean de Poitiers, il tient sa cuisse presqu'à l'horizontal, comme si le peintre voulait assimiler la monture à un siège impérial. Ces cavaliers poitevins sont parfois en train d'écraser un être humain difforme, ce qui est le cas à Cressac, les érudits en ont déduit un rapprochement typologique entre le cavalier impérial et le Christ vainqueur du mal. 
Figure 6

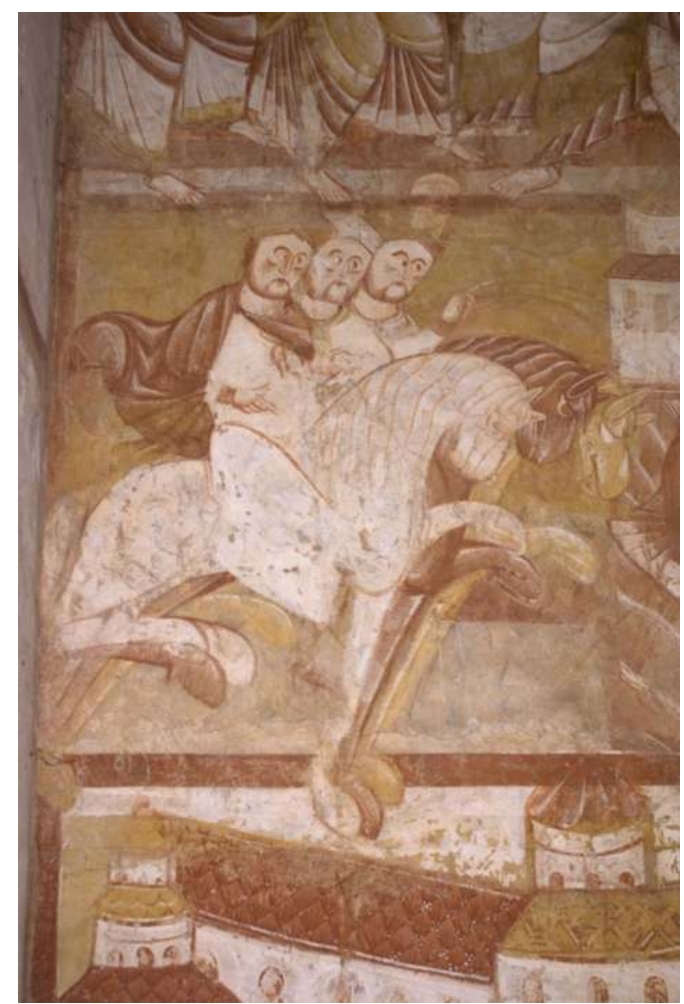

Nohant-Vic, église prieurale de Vic, arc triomphal. La Chevauchée des rois mages.

Phot. Ricarrère, Pascal. (c) Pascal Ricarrère.

Le cheval n'est pas associé au Christ, le roi céleste, tout au moins, au cours de sa vie terrestre. C'est l'âne qui porte Marie et Jésus lors de la Fuite en Égypte et c'est sur une ânesse, accompagnée de son ânon, que le Christ fait son entrée triomphale à Jérusalem. Entretemps, il voyage à pied. Le choix de cet animal est compréhensible. En effet, l'âne constitue dans l'Ancien Testament la monture des personnes de qualité et des juges, tandis que le cheval est réservé à la guerre. Le Christ, futur roi et juge, suit les usages de l'Ancienne Loi de manière à l'accomplir. Ce n'est qu'après l'avènement de la Nouvelle Loi, c'est-à-dire après sa mort et sa résurrection, que le cheval remplace l'âne auprès du Christ dans l'iconographie. La crypte de la cathédrale d'Auxerre (Yonne) présente une telle image qui s'avère exceptionnelle, voire unique. Elle est inspirée de l'Apocalypse de Jean $^{16}$. Le Christ s'avance sur un cheval blanc au pas. Il est au centre d'une vaste croix gemmée et cantonnée de quatre anges à cheval. Le peintre a illustré les versets XIX, 11 et 14: "Puis je vis le ciel ouvert et il parut sur un cheval blanc... Les armées du ciel le suivaient sur des chevaux blancs, vêtues de fin lin, blanc et pur ». Le moment représenté correspond à la victoire et le triomphe du Christ et de l'Église sur les forces du mal. À Auxerre, la scène introduit le fidèle à la contemplation de l'image ultime: la Maiestas Domini.

\section{Le cheval et son cavalier, outil de moralisation}

À travers l'image romane, l'Église a notamment cherché à galvaniser, édifier et moraliser les clercs et les fidèles. Le thème du calendrier des mois qui a été abondamment utilisé 
aussi bien Italie qu'en France présente souvent le mois de mai sous la forme d'un cavalier. De très nombreuses images le figurent plus précisément sous les traits d'un jeune homme chevauchant, un végétal à la main.

Figure 7

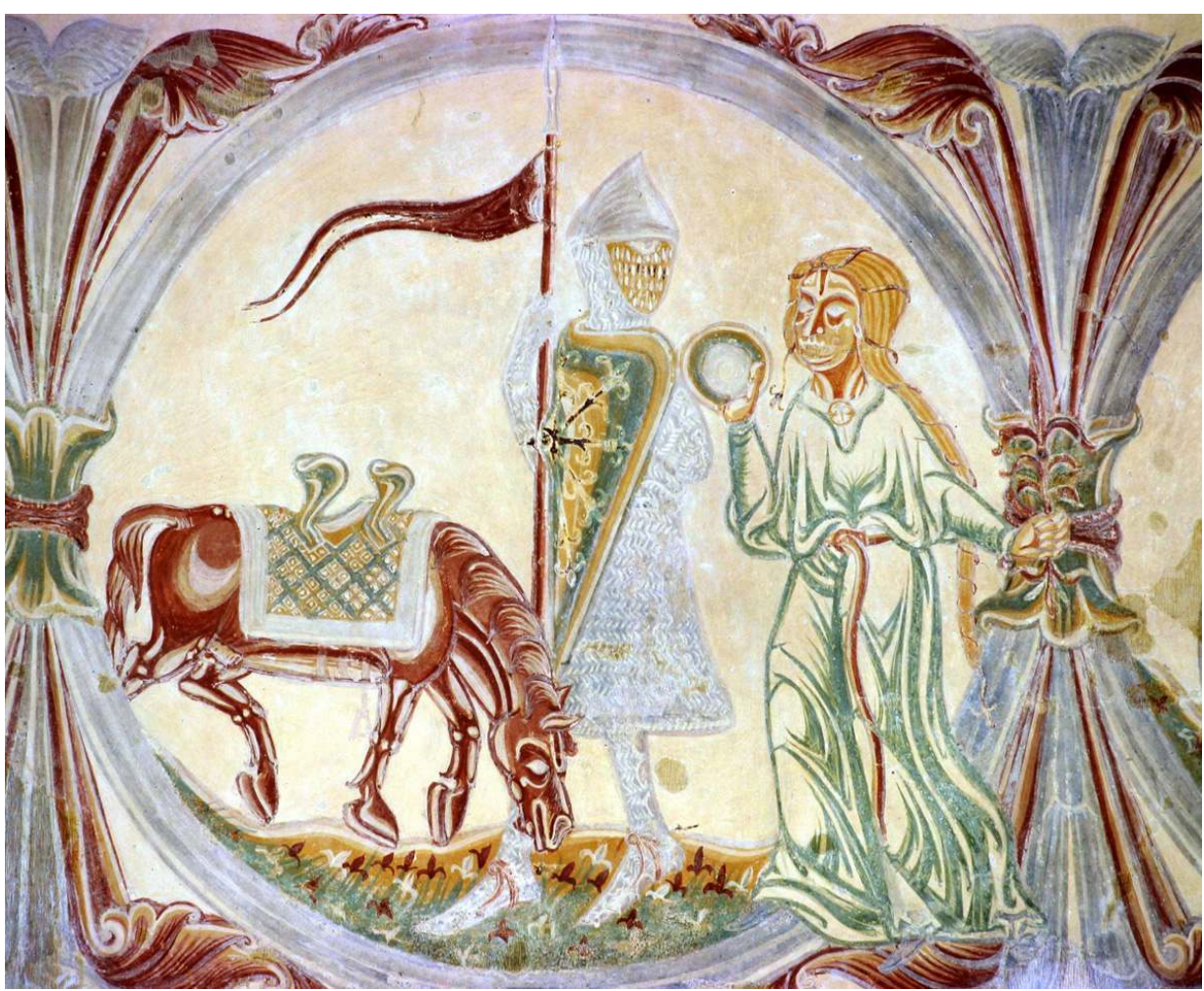

Pouzauges, église paroissiale de Vieux-Pouzauges, nef. Le Calendrier des mois, détail, le mois de mai. Phot. Giraud, Patrice. (c) Conseil régional des Pays de la Loire, inventaire général, 1995.

11 L'avertissement de ne pas céder à la tentation est peu explicite et l'image n'a pas paru suffisante à Pouzauges (Vendée). Le peintre y a retravaillé le thème du jeune cavalier pour le transformer en une image plus complexe (fig. $\mathbf{n}^{\circ} \mathbf{7}$ ). Un chevalier banneret s'apprête à monter sur son destrier pour partir à la guerre. Il porte sa longue cotte de mailles et son heaume. Il tient en main sa bannière et son écu. Il détourne la tête vers une jeune fille, reconnaissable à ses longs cheveux tressés qui l'interpelle. Celle-ci est peinte à cheval sur le cadre du médaillon, comme si elle entrait de manière concrète dans l'espace $\mathrm{du}$ chevalier. Elle lui présente un miroir, objet symbolique de la luxure ou de la mort ${ }^{17}$. L'avertissement est une mise en garde contre la mort adressée au chevalier, fort de sa jeunesse printanière et de sa force. Le chrétien, tel le chevalier, ne doit pas se laisser séduire par les beautés du monde terrestre, mais il doit au contraire s'en prémunir pour éviter le péché et la mort éternelle. À la collégiale Saint-Julien de Brioude (Haute-Loire), le chevalier et son cheval ont été renversés par une cause inconnue du spectateur. Un soldat à pied se tient debout à côté d'eux et les désigne de la main droite, l'index tendu. Une auréole dorée entoure sa tête casquée. Il est probable que soit représentée ici la chute de l'orgueilleux. La peinture murale est sans doute l'une de ces nombreuses images tirées du célèbre poème, Psychomachie, de Prudence. Ce Combat des vices et des vertus eut une très grande popularité au Moyen Âge. 


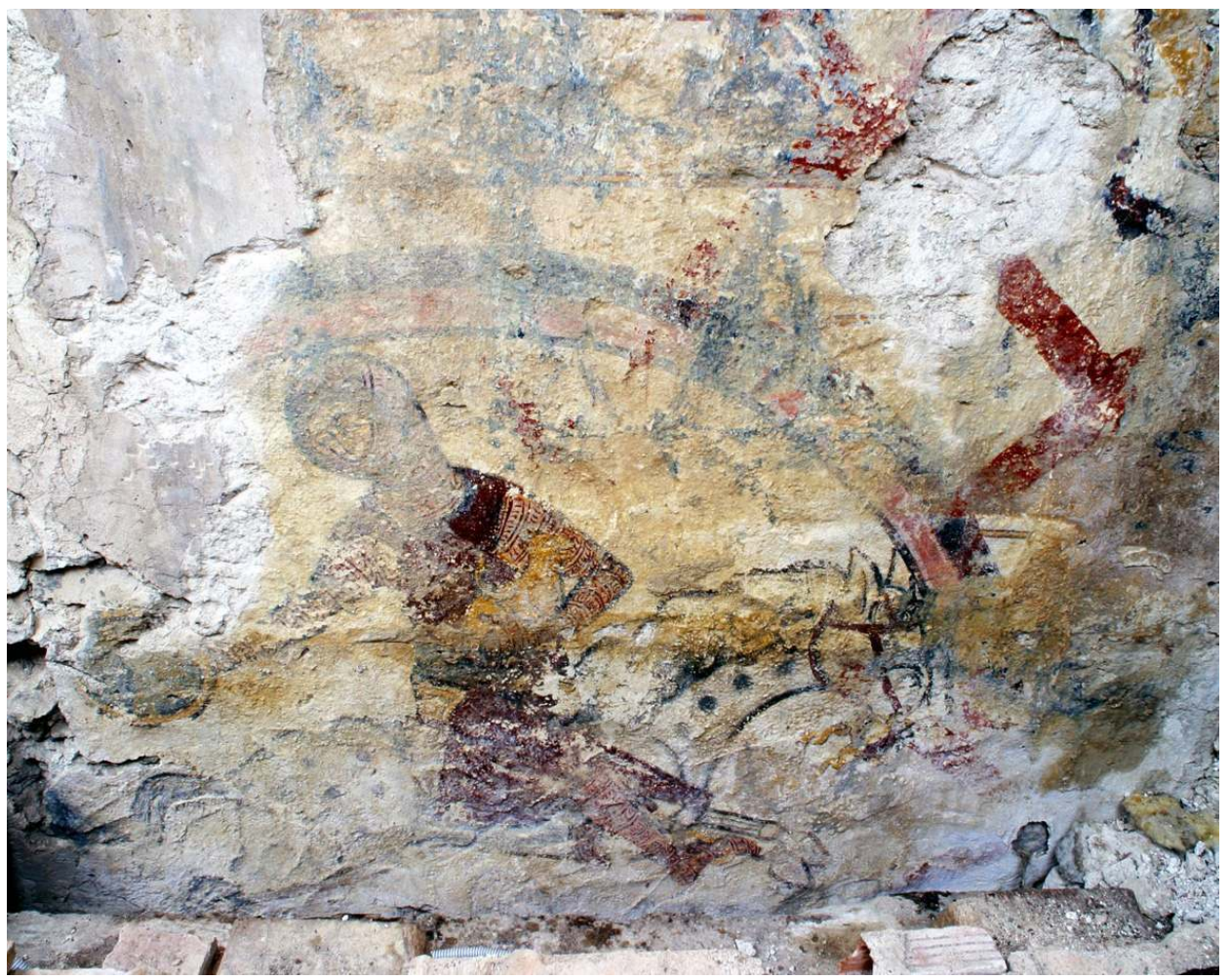

Brée, manoir de la Grande-Courbe, mur pignon sud. La Chasse au tigre, détail : le cavalier emportant le petit tigre et jetant un miroir.

Phot. Giraud, Patrice. (c) Conseil régional des Pays de la Loire, inventaire général, 1994.

12 Un autre texte antique eut également une belle renommée médiévale. Les Étymologies d'Isidore de Séville inspirèrent les Bestiaires médiévaux. L'histoire du tigre (fig. $\left.\mathbf{n}^{\circ} \mathbf{8}\right)$ a été peinte en deux médaillons au XIII ${ }^{\mathrm{e}}$ siècle dans le manoir de la Grande-Courbe à Brée (Mayenne). Le premier présente le tigre autour d'un miroir et le second un chevalier sur son cheval au galop. Il tient un petit tigre dans son bras gauche et jette un miroir de sa main droite. Là-aussi, l'avertissement moral fait au chrétien est de ne pas se laisser abuser par les apparences du monde terrestre, comme la mère tigre l'est par son reflet dans le miroir croyant y voir son petit, au risque de perdre son bien et son âme. 


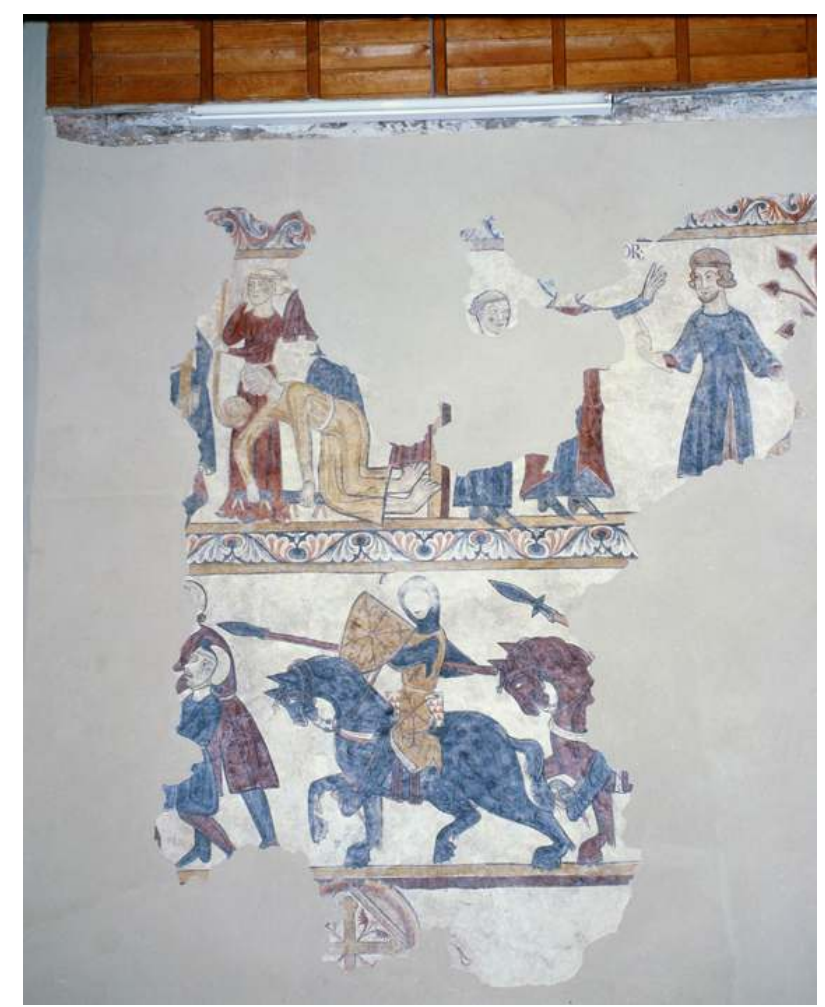

Neau, église prieurale, chœur, mur sud, partie centrale. Scènes du cycle dédié à saint Vigor. Phot. Giraud, Patrice. (c) Conseil régional des Pays de la Loire, inventaire général, 1980.

C'est une affaire beaucoup plus prosaïque qui a sans doute motivé la réalisation au XIII siècle de la peinture murale qui a été découverte dans l'église prieurale de Neau (Mayenne). Le chœur avait été orné de l'histoire de saint Vigor, missionnaire et évêque en Normandie, à qui est dédié l'édifice de culte. Parmi les nombreux miracles figurait l'épisode du comte Bertulf (fig. $\left.\mathbf{n}^{\circ} \mathbf{9}\right)$. Ce dernier est un mauvais personnage, car il spoliait non seulement les pauvres, mais aussi l'Église en dévastant ses terres. La mort fut son châtiment. Les hasards de la conservation permettent de voir aujourd'hui un chevalier sur son cheval pommelé avancer au pas et poussant devant lui des hommes à pied manifestement effrayés. Ce chevalier était suivi d'autres. Cette représentation est placée au premier registre du mur sud du chœur, à une place de choix et visible de tous. L'abbaye d'Évron, dont le prieuré de Neau dépendait, avait eu peu de temps avant la réalisation de cette peinture murale de graves démêlés avec un seigneur local à propos de droits d'usage. Leur réconciliation eut lieu en 1229 dans l'église de Neau ${ }^{18}$. Selon l'objectif des moines, le décor, peint au milieu du XIII ${ }^{\mathrm{e}}$ siècle, devait vraisemblablement être considéré comme une mise en garde aux aristocrates locaux qui contesteraient de nouveau les donations faites aux religieux par leurs parents.

\section{Une image exceptionnelle}

Nous achèverons notre étude sur une image exceptionnelle à plus d'un titre. La chapelle basse, souvent désignée comme une crypte, de l'église Notre-Dame de Montmorillon 
(Vienne), est dédiée à sainte Catherine. Elle conserve un Agneau mystique dont les formes sont manifestement inspirées de celles du cheval (fig. $\left.\mathbf{n}^{\circ} \mathbf{1 0}\right)$.

Figure 10

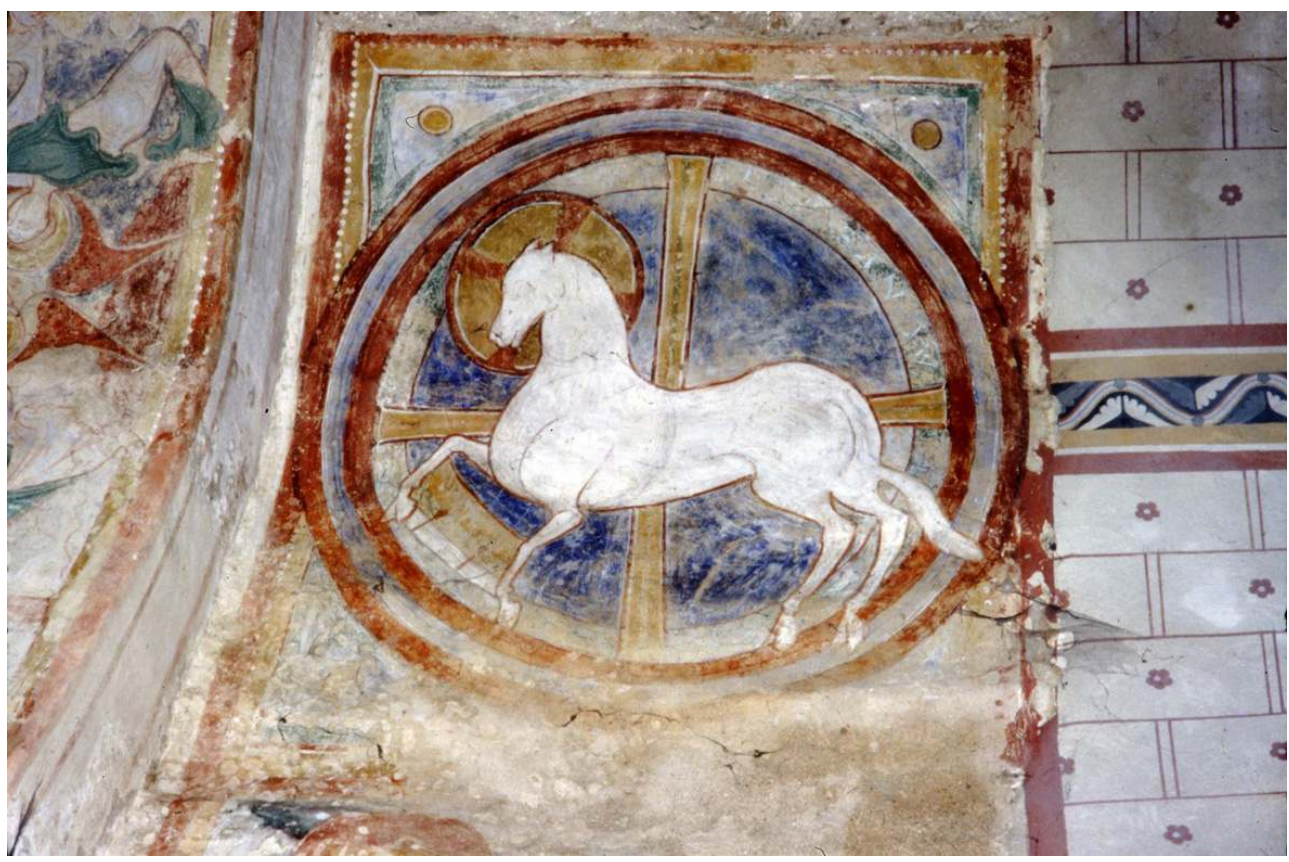

Montmorillon, église paroissiale Notre-Dame, crypte Sainte-Catherine, voûte. L'Agneau mystique.

Phot. Davy, Christian. ( C Christian Davy.

15 L'identification de l'Agneau mystique ne fait aucun doute grâce à la présence du nimbe crucifère, de l'inscription, lacunaire ${ }^{19}$, de la croix sur laquelle se détache l'Agneau, ainsi que la présence de Vieillards de l'Apocalypse. Cependant, le peintre a donné des formes assurément équines à son agneau avec des pattes élancées, un poitrail bombé, une croupe élégante et une longueur et une proportion de corps correspondant mieux à un cheval de luxe qu'à une brebis. Les sabots fendus et la taille réduite des oreilles constituent les seuls éléments correspondant à la race ovine. La représentation de l'Agneau provient de la deuxième vision préparatoire de l'Apocalypse $(\mathrm{V}, 6-8)$ : « ...et au milieu des vieillards, un Agneau était debout... ». Le livre qu'il tient entre les pattes avant est mentionné dès le verset 7 , mais il est ici représenté fermé avec un double fermoir et non avec les sept sceaux. Différents chevaux blancs sont mentionnés dans le texte de l'Apocalypse. Le premier d'entre eux est monté par l'ange à l'arc après l'ouverture du premier sceau (VI, 2). Son image est connue en peinture murale romane avec les représentations de Méobecq (Indre) et de Saint-Hilaire de Poitiers (Vienne). Les autres apparaissent au chapitre XIX qui évoque le triomphe du Christ et de l'Église, renvoyant à la peinture murale de la crypte de la cathédrale d'Auxerre évoquée précédemment dans la communication. Le commanditaire du décor peint à Sainte-Catherine de Montmorillon a manifestement tenu à combiner les deux références. Contrairement à son homologue auxerrois qui a fait développer l'idée du triomphe précédant celle du règne éternel après la fin des temps, le commanditaire poitevin a préféré insister sur l'idée de rédemption. En effet, l'équipe du Corpus des inscriptions de la France médiévale rapproche les fragments d'inscription, conservés autour du corps de l'agneau, du texte de l'évangile de Jean I, $29^{20}$ qui évoque la rémission des péchés. Cette orientation correspond à l'image essentielle du décor peinte 
au cul de four de l'abside : la représentation de l'incarnation du Christ nécessaire à cette rédemption sous les traits de la Vierge à l'Enfant.

\section{NOTES}

1. - Le Bestiaire de Pierre de Beauvais énumère les caractères qui lui permettent de le comparer au Christ alors que le verset 13 du psaume 91 (90) est le plus souvent cité pour assimiler le lion au mal : Tu marcheras sur le lion et sur l'aspic, tu fouleras le lionceau et le dragon.

2. - PICHOT, Daniel. "Cheval et société : l'exemple du Bas-Maine (IX ${ }^{e}-X I I{ }^{e}$ siècle) ». Dans Charpania. Mélanges offert à Jacques Charpy. 1991, p. 403-411; repris dans La Mayenne. Archéologie, histoire, 1992, $\mathrm{n}^{\circ}$ 15, 51-61.

3. - DESCHAMPS, Paul. «Combats de cavalerie et épisodes des Croisades dans les peintures murales du XII ${ }^{\mathrm{e}}$ et XIII ${ }^{\mathrm{e}}$ siècles ». Dans Orientalia Christiana périodica (Mélanges offerts à Guillaume de Jerphanion), 1947, v. 13, p. 454-474. On attend la publication de la thèse d'Esther Dehoux sur les saints chevaliers.

4. - Ill. dans : DAVY, Christian. La peinture murale romane dans les pays de la Loire. L'indicible et le ruban plissé. Laval : SAHM, 1999, p. 347.

5. - Histoire anonyme de la première croisade. Éditée et publiée par Louis Bréhier. Paris, 1924, p. 151-161.

6. - Voir la bibliographie dans : DAVY, Christian. «Les peintures murales romanes de la chapelle

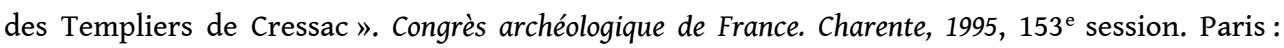
1999, p. 171-177. Voir aussi depuis : CURZI, Gaetano. La pittura dei Templari. Silvana Editoriale, 2002.

7. - Ill. dans : FAVREAU, Robert, JEANNEAU, François, RIOU, Yves-Jean et alii. Saint-Savin. L'abbaye et ses peintures murales. Poitiers : CPPPC, 1999, p. 104.

8. - FLORI, Jean. «Encore l'usage de la lance... La technique du combat chevaleresque vers l'an 1100 ». Cahiers de civilisation médiévale, 1988, t. 123, p. 213-240.

9. - Voir les différentes interventions sur ce thème : Actes des journées d'étude Le décor peint dans la demeure au Moyen Âge. Angers, 15-16 novembre 2007, voir le site: WWW.cg49.fr/culture/ peintures_murales/journees_etudes/journees_etudes.asp.

10. - PICHOT, Daniel. "Cheval et société : l'exemple du Bas-Maine (IX ${ }^{\mathrm{e}}$-XIII ${ }^{\mathrm{e}}$ siècle) ». Dans Charpania. Mélanges offert à Jacques Charpy. 1991, p. 403-411; repris dans La Mayenne. Archéologie, histoire, 1992, $\mathrm{n}^{\circ}$ 15, 51-61 ; BOURGAIN, Pascale. « Pratiques de l'équitation au Moyen Âge dans les textes littéraires ». Actes en ligne du colloque Les patrimoines de l'équitation française. Saumur, 7-9 décembre 2011. http://insitu.revues.org/9721.

11. - Située à Chinon.

12. - Illustration dans: TOULIER, Christine. Canton de Chinon. Indre-et-Loire. [S.l.] : Syndicat intercommunal du Val de Vienne (Coll. Images du patrimoine, n 81), 1990, p. 26.

13. - Je remercie chaleureusement Fabienne Audebrand, du service des Monuments historiques, DRAC du Centre, de ses remarques techniques qui m'ont ouvert les yeux sur un monde inconnu.

14. - Illustrations dans : DAVY, Christian. Op. cit. p. 119.

15. - Un fragment d'inscription donne le nom de Constantin pour l'un d'eux. Illustration dans : DEMUS, Otto. La peinture murale romane. Paris : Flammarion, 1970, pl. XLV. 
16. - Illustration dans : «Peindre à Auxerre au Moyen Âge, IX - XIVe siècle ». Sous la direction de Christian Sapin. Paris : CEM d'Auxerre et CTHS, 1999, p. 227.

17. - L'allusion à la mort est manifeste si l'on pense aux nombreuses représentations postérieures où le masque de la mort est parfois placé dans le miroir.

18. - HILLION, Yannick. Le cartulaire d'Évron. Thèse de $3^{\mathrm{e}}$ cycle, Université de Rennes II, 1977, charte 109.

19. - Les mots Agnus Dei sont lisibles et restituables.

20. - CORPUS DES INSCRIPTIONS DE LA FRANCE MÉDIÉVALE. Poitou, 1975, p. 53. « Ecce agnus Dei ecce qui tollit peccatum mundi ».

\section{RÉSUMÉS}

Le cheval est un animal rencontré de manière suffisamment régulière dans la peinture murale des $\mathrm{XI}^{\mathrm{e}}$-XIII ${ }^{\mathrm{e}}$ siècles en France pour récolter dans une certaine mesure de précieuses informations sur son état et le matériel nécessaire à sa chevauchée. Les représentations du cheval et son cavalier sont non seulement un reflet, voire un miroir, de la société féodale, mais aussi un outil de moralisation.

The horse is an animal met in a sufficiently regular way in the mural painting of the 11 th $-13^{\text {th }}$ centuries in France to collect to a certain extent invaluable information on its state and the hardware requirement with its ride. The representations of the horse and its rider are not only one reflection, even mirror, of the feudal society, but also a tool of raising the moral standard.

INDEX

Mots-clés : peinture murale, Moyen Âge, cheval, programme iconographique, décor religieux, décor profane

\section{AUTEUR}

\section{CHRISTIAN DAVY}

chercheur au service du patrimoine de la Région des Pays de la Loire

christian.davy@paysdelaloire.fr 